\title{
Cytogenetics and reproduction of sheep with multiple centric fusions (Robertsonian translocations)
}

\author{
A. N. Bruère and P. M. Ellis \\ Department of Veterinary Clinical Sciences, Massey University, Palmerston North, \\ New Zealand
}

\begin{abstract}
Summary. The significance of centric fusions (Robertsonian translocations) in domestic animals, with special reference to sheep, is reviewed. The mating is described of a further 856 ewes with either a normal chromosome number $2 n=54$ or carrying one or more of the three different translocations (centric fusions) $t_{1}, t_{2}$ and $t_{3}$ in various heterozygous and homozygous arrangements. Rams which were used in the matings were homozygous for one of the translocation chromosomes $(2 n=52)$, double heterozygotes $(2 n=52)$, triple heterozygotes $(2 n=51)$ or were carriers of 4 translocation chromosomes $(2 n=50)$ and 5 translocation chromosomes $(2 \mathrm{n}=49)$.

A remarkably even distribution of segregation products was recorded in the progeny of all combinations of translocation ewes $\times$ translocation rams in those groups in which sufficient animals were available for statistical analysis. Forty-eight chromosomally different groups of animals were mated. Further, the overall fertility of the translocation sheep, measured by conception rate to first service, lambing percentage and number of ewes which did not breed a lamb, was not significantly different from New Zealand national sheep breeding data. In some groups the poorer reproductive performance could be explained by the age structure of the flock and inbreeding depression, which probably affected the performance of some animals.

Sheep with progressively decreasing chromosome numbers, due to centric fusion, $2 n=50,2 n=49$ and $2 n=48$, are reported. The $2 n=48$ category represents a triple homozygous ewe and a triple homozygous ram and is the first report of the viable evolution of such domestic animals. Less than $1 \%$ of phenotypically abnormal lambs were recorded in a total of 1995 progeny born over 10 years. It is now considered that there is little or no evidence to suggest that centric fusions in a variety of combinations affect the total reproductive fitness of domestic sheep. It is suggested that future research should be more actively directed to understanding their genetic significance.
\end{abstract}

\section{Introduction}

A high frequency of centric fusions (Robertsonian translocations) has been described in several species of domestic animals (Bruère, 1974b). For this reason it has become increasingly important to understand both their effects on fertility and their role, if any, on the development of these species. Because of the slow generation period of our domestic animals the accumulation of data that can be used for these purposes may take many years. However, there is mounting evidence that in some species centric fusions do not have a significant effect on total reproductive fitness. This has been demonstrated from accumulated breeding data and from normal segregation ratios which result from the products of crossing sheep that have centric fusions in various mating categories (Bruère, 1974a; Bruère \& Chapman, 1974; Bruère, 1975; 
Long, 1978b). It is believed that the normal reproductive performance and the balanced segregation ratios reported in these breeding data were produced because only balanced gametes took part in fertilization although aneuploid secondary spermatocytes were recorded at significant levels in all heterozygous rams $\left(t_{1}, 5.63 \% ; t_{2}, 4.5 \% ; t_{3}, 9 \cdot 2 \%\right.$ (Chapman \& Bruère, 1975); 6.1\% in $t_{1}$, only (Long, 1978a)).

Although it is possible that centric fusions may form spontaneously in domestic sheep and domestic cattle it is believed that the majority of translocation-carrying animals so far found from population surveys has been derived from a common ancestor (Gustavsson, 1969; Bruère, Chapman, Jaine \& Morris, 1976). It has also been suggested that their increase in most domestic species has been slow but positive, due either to a slight heterozygous advantage (Bruère, Evans, Burtenshaw \& Brown, 1978) or to the chance selection of a carrier animal as a sire in a breeding programme that involved a small population of animals (John, 1976).

The mechanism by which centric fusions are formed and their significance is uncertain (Bruère, Zartman \& Chapman, 1974; John \& Freeman, 1975; Kurnit, Brown \& Maio, 1978) but it is generally believed that their formation does not mean that significant genetic material is lost. However, the development of an animal population with a number of different centric fusions may produce genetic differences which reduce fertility when such animals are mated back to others of the original or normal karyotype, as demonstrated in the $F_{1}$ of the tobacco mouse mated to the domestic mouse (Gropp, Tettenborn \& Von Lehmann, 1970; Cattanach \& Moseley, 1973). A comparable situation to the tobacco mouse has also been reported for the Apennine mouse with a 22 chromosome karyotype (Capanna, Gropp, Winking, Noack \& Civitelli, 1976).

Reduced fertility in the tobacco mouse $x$ domestic mouse is probably due to the genetic differences between the species of animals involved (Cattanach \& Moseley, 1973; Cattanach, 1978). Similarly, the frequency of trisomy, with a decrease in viability of progeny in certain strains of translocation-carrying mice may be attributable to genotype influences (White, Tjio, Van de Water \& Crandall, 1974a). Although double translocation heterozygotes have been reported as occurring randomly in sheep (Bruère et al., 1976), there is no information on the effect of rapidly combining three centric fusions or Robertsonian translocations into one animal in the homozygous state. Further, it could be inferred from the example of the tobacco mouse that the effects of centric fusions in the heterozygous state tend to be either additive or at least increased. Individual centric fusions in tobacco mice produce quite different levels of aneuploid secondary spermatocyte frequency, ranging from 6.0 (T6) to 29.5 (T4) with individual variations (Cattanach \& Moseley, 1973). However high levels of aneuploidy are also consistent with high frequencies of zygotic loss (Tettenborn \& Gropp, 1970; Cattanach \& Moseley, 1973). It is therefore reasonable to propose that, since all three centric fusions of domestic sheep produce consistent levels of aneuploid secondary spermatocyte frequency in the heterozygous state (Chapman \& Bruère, 1975), in combination their effects may become apparent. For example, a triply heterozygous ram may produce a level of aneuploid spermatocyte frequency which is equal to the sum of the individual levels of singly heterozygous rams. If the same level of aneuploidy is produced in a triply heterozygous ewe then it could be proposed that mating such animals should show an obvious reduction in fertility were the unbalanced spermatocytes or oocytes to form gametes which took part in fertilization. A normal level of fertility on the other hand would strengthen our previous conclusion that aneuploid secondary spermatocytes in the sheep do not produce gametes which either participate in fertilization or at least significantly participate in fertilization.

The present report describes the results of mating rams of various translocation types with a further 856 ewes which had a normal chromosome number $2 n=54$ or were carrying one or more of the three translocations, $t_{1}, t_{2}$ and $t_{3}$, in various heterozygous and homozygous combinations (Table 1). These data, in association with the previous results from mating 862 ewes with single translocation heterozygous rams and homozygous translocation rams, present informa- 
tion on 1718 matings in which 1995 progeny were born and 1676 were analysed chromosomally. They also report for the first time the effects of rapidly breeding three different centric fusions into one animal to produce sheep in which $2 n=48$ and also present further information on the effects of centric fusions on fertility and karyotypes of domestic sheep.

\section{Materials and Methods}

\section{Animals and reproductive performance}

The breeding system and animal husbandry from which the present results were derived were the same as reported previously (Bruère, 1974a, 1975). Because of the slow generation time of 2 years for sheep, it was frequently necessary, in order to increase the data, to use ewe lambs for breeding. The breeding performance for such animals is lower than for mature sheep and so the fertility results from these sheep were not used in the comparisons of reproductive performance. Comparisons of reproductive performance were obtained by the procedure described by Bruère $(1974 a, 1975)$. The reference data were: (1) conception rate to first service $\left(C_{R}\right) ;(2)$ number of dry ewes, i.e. ewes mated but not producing a lamb $\left(E_{D M}\right) ;(3)$ number of ewes mated $\left(E_{M}\right) ;(4)$ number of lambs born dead or alive $\left(\mathrm{L}_{\mathrm{BM}}\right)$; and $(5)$ number of lambs weaned $\left(\mathrm{L}_{\mathrm{wM}}\right)$. The difference between (2) and (3) was used as a measure for conception rate and embryonic survival. The difference between (4) and (5) was used as a measure of reproductive rate and can also be a useful indicator of inbreeding depression (Lax \& Brown, 1968).

\section{Cytogenetics, segregation ratios and mating groups}

The same methods of blood collection, leucocyte culture and harvesting were used throughout the 10-year period of study. The same G-band identification method as described previously was used (Bruère, 1975). In addition the silver staining technique for showing NORs (nucleolus organizer regions) as described by Henderson \& Bruère (1977) was used to identify the translocation chromosome in some of the animals with several translocation chromosomes. The progeny of some mating groups were not analysed chromosomally because the ewes were also used for a student teaching exercise, which interfered with lamb survival. Only the total lambs born from these data were used for information on reproductive performance.

The chances of producing sheep homozygous for all three translocations decreased as the number of translocations of the various heterozygotes and homozygotes increased. For this reason no selection was made against the usual undesirable traits which occur in animal breeding, or for the small size of some sheep used in these experiments. Selection against undesirable traits and for low body weight are common criteria used to maintain sound breeding performance in New Zealand sheep. Therefore it is likely that the reproductive performance results described here are biased against the translocation-carrying sheep. For a general comparison of reproductive performance, records were compared with those of commercial flocks of sheep husbanded in similar circumstances on the University farms. Also for a general comparison the annual performance of the National sheep flock was used.

In the present data 48 different chromosome combinations were recorded. However, as many of these were too small for significant analysis on their own they have been recorded in main mating groups of chromosome categories as described in Table 1. In some instances special mating groups have been extracted from these main groups and the results discussed separately.

Without identification by special staining techniques $t_{1}$ and $t_{2}$ are indistinguishable so that when both chromosomes occurred in one animal and were not identified the symbol $t_{x}$ was used. Thus in some data $t_{x} t_{x}$ could be either a homozygote or a double heterozygote. Similarly, in other tables and in the text where, for clarity, some data have been combined, the $t$ symbols have been 
Table 1. Broad summary of main translocation groups (1975-1978) showing numbers and types of sheep mated and lambs analysed chromosomally

\begin{tabular}{|c|c|c|c|}
\hline \multicolumn{2}{|c|}{ Karyotype of mating category } & \multirow{2}{*}{$\begin{array}{l}\text { No. of ewes } \\
\text { mated }\end{array}$} & \multirow{2}{*}{$\begin{array}{c}\text { No. lambs } \\
\text { analysed } \\
\text { chromosomally }\end{array}$} \\
\hline Rams & Ewes & & \\
\hline $51, x y, t_{1} t_{2} t_{3}$ & $54, \mathrm{xx}$ & 54 & 53 \\
\hline $51, x y, t_{1} t_{2} t_{3}$ & $53, \mathrm{xx}, \mathrm{t}_{1}, 53, \mathrm{xx}, \mathrm{t}_{2}, 53, \mathrm{xx}, \mathrm{t}_{3}$ & 126 & 128 \\
\hline $51, x y, t_{1} t_{2} t_{3}$ & $\begin{array}{l}52, \mathrm{xx}, \mathrm{t}_{1} \mathrm{t}_{3}, 52, \mathrm{xx}, \mathrm{t}_{1} \mathrm{t}_{2} \\
52, \mathrm{xx}, \mathrm{t}_{2} \mathrm{t}_{3}, 52, \mathrm{xx}, \mathrm{t}_{\mathrm{x}} \mathrm{t}_{\mathrm{x}} \mathrm{t}^{*}\end{array}$ & 98 & 80 \\
\hline $51, \mathrm{xy}, \mathrm{t}_{1} \mathrm{t}_{2} \mathrm{t}_{3}$ & $51, \mathbf{x x}, \mathrm{t}_{\mathbf{x}} \mathrm{t}_{\mathbf{x}} \mathrm{t}_{2}^{*}$ & 23 & 14 \\
\hline $51, x y, t_{1} t_{2} t_{3}$ & $\begin{array}{l}52, \mathrm{xx}, \mathrm{t}_{1} \mathrm{t}_{1}, 52, \mathrm{xx}, \mathrm{t}_{2} \mathrm{t}_{2} \\
52, \mathrm{xx}, \mathrm{t}_{3} \mathrm{t}_{3}\end{array}$ & 70 & 65 \\
\hline \multirow{3}{*}{$\begin{array}{l}51, x y, t_{1} t_{2} t_{3} \\
52, x y, t_{1} t_{2} \\
52, x y, t_{1} t_{3}\end{array}$} & $50, x x, t t t t, 49, x x, t_{1} t_{1} t_{2} t_{3} t_{3}$ & 5 & 4 \\
\hline & $53, \mathrm{xx}, \mathrm{t}_{1}, 53, \mathrm{xx}, \mathrm{t}_{2}, 53, \mathrm{xx}, \mathrm{t}_{3}$ & 122 & 116 \\
\hline & $\begin{array}{l}54, \mathrm{xx}, 53, \mathrm{xx}, \mathrm{t}_{1}, 53, \mathrm{xx}, \mathrm{t}_{2} \\
53, \mathrm{xx}, \mathrm{t}_{3}, 52, \mathrm{xx}, \mathrm{t}_{1} \mathrm{t}_{1}, 52, \mathrm{xx}, \mathrm{t}_{1} \mathrm{t}_{3}\end{array}$ & 78 & 0 \\
\hline \multirow{11}{*}{$\begin{array}{l}52, x y, t_{2} t_{2} \\
52, x y, t_{1} t_{1} \\
52, x y, t_{2} t_{2} \\
52, x y, t_{3} t_{3} \\
52, x y, t_{1} t_{1} \\
52, x y, t_{2} t_{2} \\
53, x y, t_{3} t_{3} \\
49, x y, t_{1} t_{2} t_{2} t_{3} t_{3} \\
50, x y, t_{1} t_{2} t_{3} t_{3} \\
53, x y, t_{2} \\
53, x y, t_{3}\end{array}$} & $53, \mathrm{xx}, \mathrm{t}_{2}$ & 25 & 30 \\
\hline & $52, \mathrm{xx}, \mathrm{t}_{1} \mathrm{t}_{1}, 52, \mathrm{xx}, \mathrm{t}_{2} \mathrm{t}_{2}$ & 33 & 29 \\
\hline & $52, \mathrm{xx}, \mathrm{t}_{3} \mathrm{t}_{3}$ & & \\
\hline & & & \\
\hline & $52, \mathbf{x x}, \mathrm{t}_{1} \mathrm{t}_{3}, 52, \mathrm{xx}, \mathrm{t}_{1} \mathrm{t}_{2}$ & 86 & 79 \\
\hline & $53, x_{x}, t_{1}$ & & \\
\hline & $54, x x$ to $49, x x, t_{1} t_{1} t_{2} t_{3} t_{3}$ & 49 & 44 \\
\hline & & & \\
\hline & $53, \mathrm{xx}, \mathrm{t}_{2}$ & 28 & 27 \\
\hline & $54, \mathrm{xx}$ & 59 & 72 \\
\hline & Totals & 856 & 741 \\
\hline
\end{tabular}

left unidentified and the number of animals with both the expected and observed chromosome numbers only are given.

\section{Results}

\section{Segregation patterns}

As the number of translocation chromosomes carried in the heterozygous state by individual animals increases, the number of segregation products among the progeny also increases. Therefore in the present data because of the small number of products in some groups not all the results could be analysed. However, sufficient data are available from the majority of the different mating combinations to suggest that the translocations segregate normally among progeny even when both parents are heterozygous for all three translocations. Tables 2, 3 and 4 demonstrate this point and show the segregation patterns from various matings.

In Table 2(a) a significant deficit of $t_{2}$ progeny was produced. From the total numbers of animals in the mating group it is perhaps premature to suggest a selective disadvantage for the $t_{2}$ chromosome. A similar significant deficit of $t_{2}$ ewe lambs was reported in a previous set of data (Bruère, 1974a) but additional data (Bruère, 1975) nullified such a suggestion. In none of the other data involving the $t_{2}$ chromosome was such a deficit recorded.

In Table 3 the individual data from each mating group are few but each still shows the clear tendency to the production of the expected proportions of chromosome products and the combination of the three groups shows no significant deviation from the expected ratio of progeny in 
Table 2. Numbers of progeny with respective chromosome number recorded from matings of triple heterozygous rams

\begin{tabular}{|c|c|c|c|c|c|c|c|c|}
\hline \multicolumn{2}{|l|}{ Matings } & \multicolumn{6}{|c|}{ Chromosome number of progeny } & \multirow[t]{2}{*}{ Total } \\
\hline & & $51, t_{1} t_{2} t_{3}$ & $52, \mathrm{t}_{1} \mathrm{t}_{3}$ & $52, \mathrm{t}_{\mathrm{x}} \mathrm{t}_{2}$ & $53, t_{x}$ & $53, \mathrm{t}_{2}$ & 54 & \\
\hline \multirow{3}{*}{ (a) $\begin{aligned} & 51, x y, t_{1} t_{2} t_{3} \\
& \times 54, x x\end{aligned}$} & $\begin{array}{l}\text { Expected } \\
\text { numbers }\end{array}$ & 6.625 & 6.625 & $13 \cdot 25$ & $13 \cdot 25$ & 6.625 & $6 \cdot 625$ & 53 \\
\hline & $\begin{array}{l}\text { Observed } \\
\text { numbers }\end{array}$ & 8 & 3 & 16 & 12 & 0 & 14 & 53 \\
\hline & & $50, \mathrm{t} \mathrm{t} \mathrm{t} \mathrm{t}$ & $51, \mathrm{t} \mathrm{t} \mathrm{t}$ & $52, \mathrm{t} \mathrm{t}$ & $53, \mathrm{t}$ & & & \\
\hline \multirow{2}{*}{ (b) $\begin{array}{r}51, \mathrm{xy}, \mathrm{t}_{1} \mathrm{t}_{2} \mathrm{t}_{3} \\
\times 52, \mathrm{xx}, \mathrm{t}_{1} \mathrm{t}_{1} \\
52, \mathrm{xx}, \mathrm{t}_{2} \mathrm{t}_{2} \\
52, \mathrm{xx}, \mathrm{t}_{3} \mathrm{t}_{3}\end{array}$} & $\begin{array}{l}\text { Expected } \\
\text { numbers }\end{array}$ & $8 \cdot 125$ & $24 \cdot 75$ & $24 \cdot 75$ & $8 \cdot 125$ & & & 65 \\
\hline & $\begin{array}{l}\text { Observed } \\
\text { numbers }\end{array}$ & 7 & 24 & 27 & 7 & & & 65 \\
\hline
\end{tabular}

(a) $\chi_{5}^{2}=14 \cdot 81 ; 0 \cdot 01<P<0.02(1: 1: 2: 2: 1: 1$ ratio $)$
(b) $\chi_{3}^{2}=$ not significant $(1: 3: 3: 1$ ratio).

Table 3. Numbers of progeny with respective chromosome number recorded from matings of triple heterozygous rams

\begin{tabular}{|c|c|c|c|c|c|c|c|}
\hline \multicolumn{2}{|l|}{ Matings } & \multicolumn{5}{|c|}{ Chromosome number of progeny } & \multirow[t]{2}{*}{ Total } \\
\hline \multirow{3}{*}{$\begin{array}{l}51, \mathrm{xy}, \mathrm{t}_{1} \mathrm{t}_{2} \mathrm{t}_{3} \\
\times 53, \mathrm{xx}, \mathrm{t}_{1}\end{array}$} & & $50, \mathrm{t} t \mathrm{tt}$ & $51, \mathrm{t} t \mathrm{t}$ & $52, \mathrm{t} \mathrm{t}$ & $53, \mathrm{t}$ & 54 & \\
\hline & $\begin{array}{l}\text { Expected } \\
\text { numbers }\end{array}$ & $2 \cdot 25$ & 9 & $13 \cdot 5$ & 9 & $2 \cdot 25$ & 36 \\
\hline & $\begin{array}{l}\text { Observed } \\
\text { numbers }\end{array}$ & 2 & 9 & 15 & 9 & 1 & 36 \\
\hline (b) $\begin{aligned} & 51, \mathrm{xy}_{1} \mathrm{t}_{1} \mathrm{t}_{2} \mathrm{t}_{3} \\
\times & \times 33, \mathrm{xx}, \mathrm{t}_{2}\end{aligned}$ & $\begin{array}{l}\text { Expected } \\
\text { numbers }\end{array}$ & 2 & 8 & 12 & 8 & 2 & 32 \\
\hline & $\begin{array}{c}\text { Observed } \\
\text { numbers }\end{array}$ & 0 & 10 & 11 & 9 & 2 & 32 \\
\hline \multirow[t]{2}{*}{ (c) $\begin{aligned} & 51, \mathrm{xy}, \mathrm{t}_{1} \mathrm{t}_{2} \mathrm{t}_{3} \\
& \times 53, \mathrm{xx}, \mathrm{t}_{3}\end{aligned}$} & $\begin{array}{l}\text { Expected } \\
\text { numbers }\end{array}$ & $3 \cdot 75$ & 15 & $22 \cdot 5$ & 15 & 3.75 & 60 \\
\hline & $\begin{array}{l}\text { Observed } \\
\text { numbers }\end{array}$ & 1 & 18 & 19 & 16 & 6 & 60 \\
\hline \multirow[t]{2}{*}{ Total } & $\begin{array}{l}\text { Expected } \\
\text { numbers }\end{array}$ & 8 & 32 & 48 & 32 & 8 & 128 \\
\hline & $\begin{array}{l}\text { Observed } \\
\text { numbers }\end{array}$ & 3 & 37 & 45 & 34 & 9 & 128 \\
\hline
\end{tabular}

$\chi_{5}^{2}=$ not significant $(1: 4: 6: 4: 1$ ratio $)$.

each chromosome group. Similar comment applies to Table 4 excejt that the number of segregation products is even more complicated but still regular and the sheep with 49 and 50 chromosomes were produced (Pl. 1, Figs 1 and 2).

Table 5 gives data additional to those reported previously (Bruère, 1975) concerning progeny of $52, x y, t_{1} t_{2}$ rams, but in this instance the ewes carried a single Robertsonian translocation where previous data were from chromosomally normal ewes. Table 6 is a record of 10 previously 
Table 4. Numbers of progeny with respective chromosome number recorded from matings of triple heterozygous rams

\begin{tabular}{|c|c|c|c|c|c|c|c|c|c|}
\hline \multicolumn{3}{|c|}{ Matings } & \multicolumn{6}{|c|}{ Chromosome number of progeny } & \multirow[t]{2}{*}{ Total } \\
\hline \multirow{3}{*}{ (a) } & \multirow{3}{*}{$\begin{array}{l}51, x y, t_{1} t_{2} t_{3} \\
\times 52, x x, t_{1} t_{2}\end{array}$} & & $49, \mathrm{t} \mathrm{t} \mathrm{t} \mathrm{t} \mathrm{t}$ & $50, \mathrm{tt} \mathrm{t}$ & $51, \mathrm{t} \mathrm{t} \mathrm{t}$ & $52, \mathrm{t} \mathrm{t}$ & $53, \mathrm{t}$ & 54 & \\
\hline & & $\begin{array}{l}\text { Expected } \\
\text { numbers }\end{array}$ & $1 \cdot 19$ & 5.94 & 11.87 & 11.87 & 5.94 & $1 \cdot 19$ & 38 \\
\hline & & $\begin{array}{l}\text { Observed } \\
\text { numbers }\end{array}$ & 2 & 9 & 12 & 8 & 4 & 3 & 38 \\
\hline \multirow[t]{2}{*}{ (b) } & $\begin{array}{l}51, x y, t_{1} t_{2} t_{3} \\
\times 52, x x, t_{1} t_{3}\end{array}$ & $\begin{array}{l}\text { Expected } \\
\text { numbers }\end{array}$ & $0 \cdot 81$ & $4 \cdot 06$ & $8 \cdot 12$ & $8 \cdot 12$ & 4.06 & 0.81 & 26 \\
\hline & & $\begin{array}{l}\text { Observed } \\
\text { Numbers }\end{array}$ & 1 & 3 & 6 & 11 & 3 & 2 & 26 \\
\hline \multirow[t]{4}{*}{ (c) } & \multirow[t]{2}{*}{$\begin{array}{l}51, x y, t_{1} t_{2} t_{3} \\
\times 52, \mathrm{t}_{3}, \mathrm{t}_{2} \mathrm{t}_{3}\end{array}$} & $\begin{array}{l}\text { Expected } \\
\text { numbers }\end{array}$ & 0.375 & 1.875 & 3.75 & $3 \cdot 75$ & 1.875 & 0.375 & 12 \\
\hline & & $\begin{array}{l}\text { Observed } \\
\text { numbers }\end{array}$ & 1 & 4 & 2 & 2 & 3 & 0 & 12 \\
\hline & \multirow[t]{2}{*}{ Total } & $\begin{array}{l}\text { Expected } \\
\text { numbers }\end{array}$ & 2.375 & 11.875 & $23 \cdot 74$ & $23 \cdot 74$ & 11.875 & $2 \cdot 375$ & 76 \\
\hline & & $\begin{array}{l}\text { Observed } \\
\text { numbers }\end{array}$ & 4 & 16 & 20 & 21 & 10 & 5 & 76 \\
\hline
\end{tabular}

$\chi_{\mathrm{s}}^{2}=$ not significant $(1: 5: 10: 10: 5: 1$ ratio).

unreported matings which show predictable segregation results which are also valuable in confirming that no deleterious genetic effect is produced by any of the three translocation chromosomes when heterozygous, homozygous or in combination with each other no matter in what combination they occur.

In addition to the above data, rams which were $49, x y, t_{1} t_{2} t_{2} t_{3} t_{3}$ and $50, x y, t_{1} t_{2} t_{3} t_{3}$ were mated to a variety of ewes from a normal chromosome number to one ewe which had 49 chromosomes and was a double translocation homozygote, heterozygote $\left(49, x x, t_{1} t_{1} t_{2} t_{3} t_{3}\right)$. In each instance normal progeny were born but the data were too small to tabulate. However, for the first time from these matings, lambs were born with 48 chromosomes: 1 ewe and 1 ram (Pl. 2, Figs 3 and 4).

\section{Reproductive performance}

Table 7 gives details of mating homozygous translocation ewes of three different translocation types and also doubly translocation heterozygous sheep of three types to a variety of chromosomally different rams. The $\mathrm{E}_{\mathrm{M}}$ (ewes mated) really refers to the same ewes mated over several seasons, for example in Table 7 (a) only $252, x x, t_{1} t_{1}$ ewes were mated in 1973 but as further animals were bred they were added to the flock. This applies to most of the data. The reproductive performance of the sheep shown in Table 7 is not as good as the previous results of mating single translocation heterozygotes (Bruère, 1974a, 1975). However, there are several possible explanations for this. (1) Non-selection of ewes for good breeding performance as happens in most commercial sheep flocks. (2) The age structure of the flock; ewes, lambs and 18 month ewes were used frequently and the lambing performance of both groups is lower than that of older ewes. (The data from ewe lambs which did not breed were excluded.) (3) A high lamb loss due to perinatal mortality (mainly dystocia); see Table 10. (4) Inbreeding depression; some of the ewes used in these experiments were more closely bred in later years of the breeding programme 

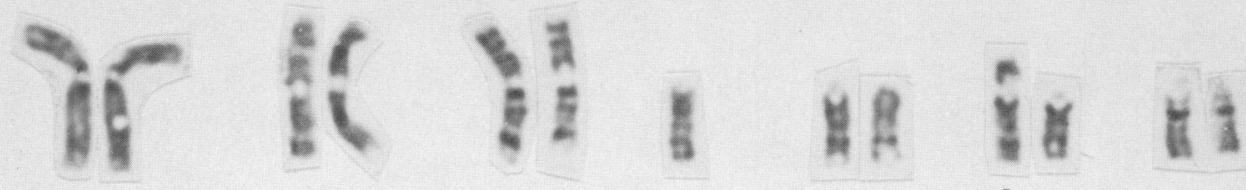

$t_{1}$

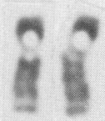

c

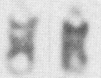

\section{M}

$M B$

7

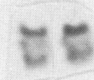

$4 x$

$t_{3} t_{3}$

$t_{2}$

$\because:$

$x: 1$

มี มี

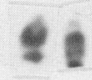

पै

6 11

$4=$

8

a 1
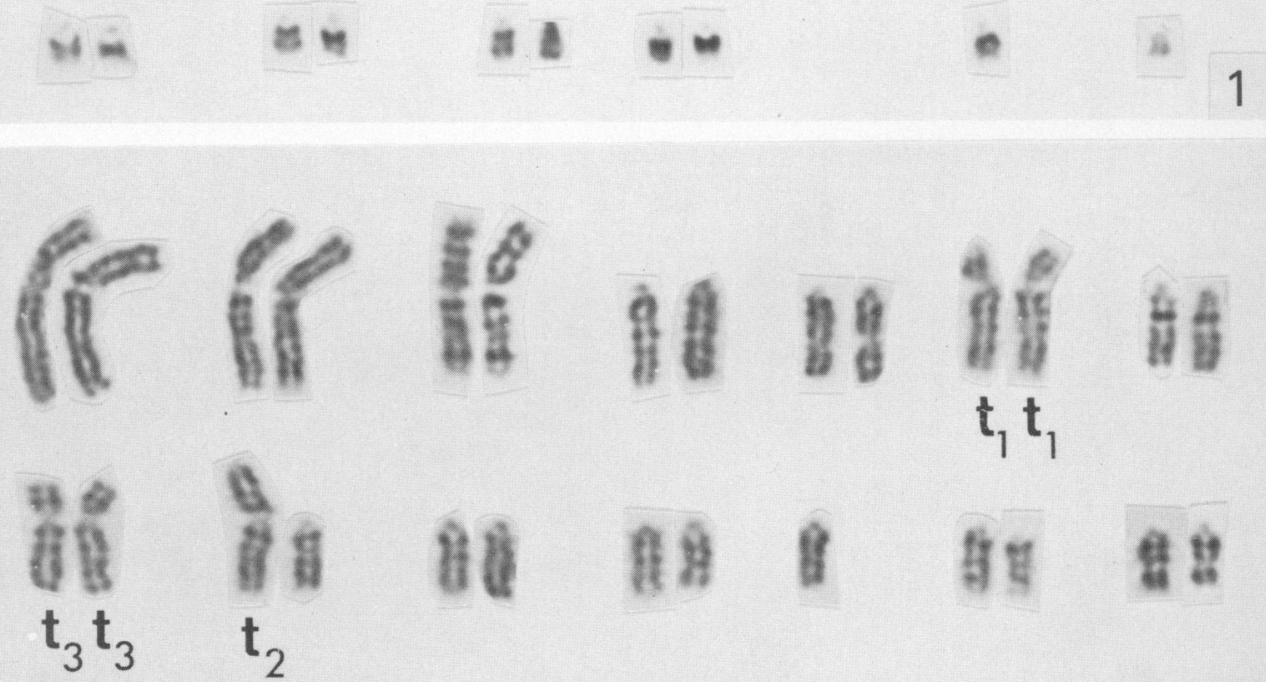

aी

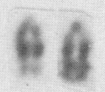

at

ำ ใด

at

f요

료

c s

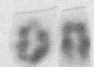

ตล

6 1

Fig. 1. The karyotype of a $50, x y, t_{1} t_{2} t_{3} t_{3}$ ram. G-banded, $\times 2000$.

Fig. 2. The karyotype of a $49, x x, t_{1} t_{1} t_{2} t_{3} t_{3}$ ewe. G-banded, $\times 2000$. 


\section{PLATE 2}

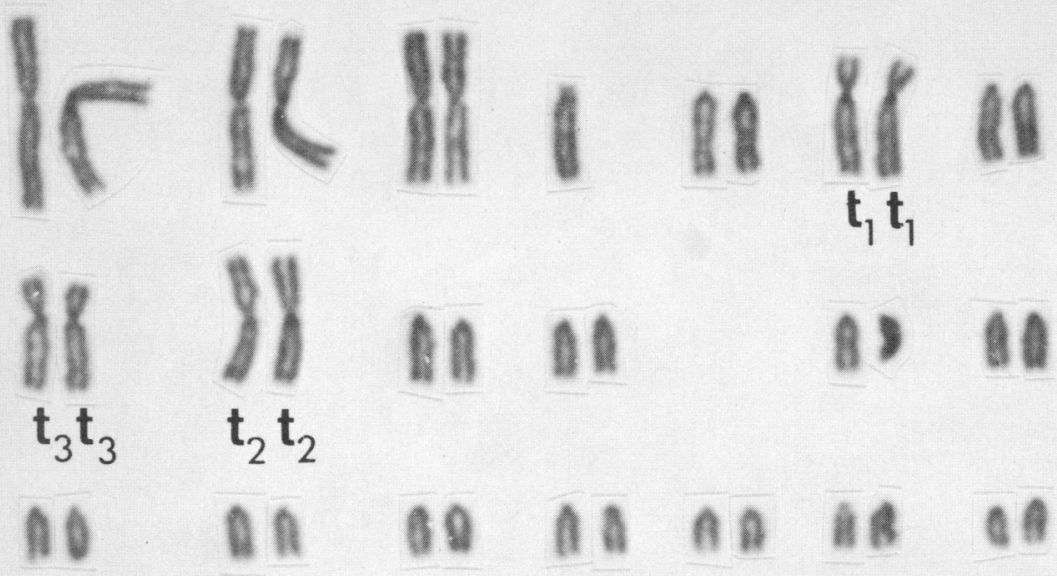

ก ก ค ด ด ถ
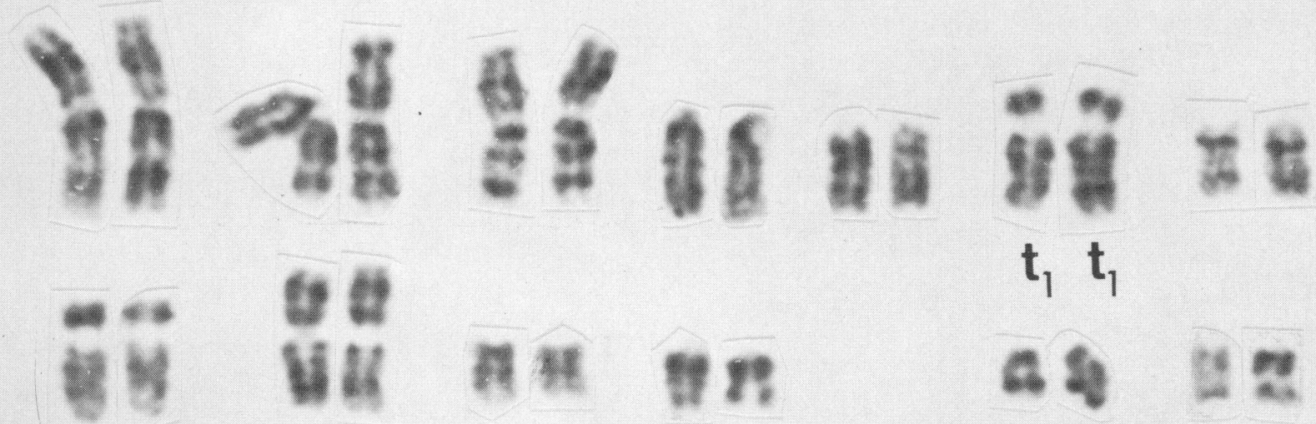

$$
t_{1} t_{1}
$$

$t_{3} t_{3}$

$t_{2} t_{2}$

तै तै

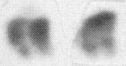

8) 14

ก $n$ is $4 * 4$

20
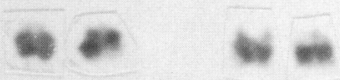

* *

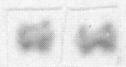

Fig. 3. The karyotype of a $48, x y, t_{1} t_{1} t_{2} t_{2} t_{3} t_{3}$ ram. Giemsa stained, $\times 2000$.

Fig. 4. The karyotype of a 48, xx, $t_{1} t_{1} t_{2} t_{2} t_{3} t_{3}$ ewe. G-banded, $\times 2000$. 
Table 5. Numbers of progeny with respective chromosome number recorded from matings of $52, x y, t_{1} t_{2}$ rams

\begin{tabular}{|c|c|c|c|c|c|c|}
\hline Matings & & & Chromosome $n$ & er of progeny & & Tota \\
\hline \multirow{3}{*}{ (a) $\begin{aligned} & 52, \mathrm{xy}, \mathrm{t}_{1} \mathrm{t}_{2} \\
& \times 53, \mathrm{xx}, \mathrm{t}_{1}\end{aligned}$} & & $51, t_{1} t_{1} t_{2}$ & $52, \mathrm{t}_{1} \mathrm{t}_{1}+52, \mathrm{t}_{1} \mathrm{t}_{2}$ & $53, \mathrm{t}_{1}+53, \mathrm{t}_{2}$ & 54 & \\
\hline & $\begin{array}{l}\text { Expected } \\
\text { numbers }\end{array}$ & $6 \cdot 375$ & $19 \cdot 125$ & $19 \cdot 125$ & 6.375 & 51 \\
\hline & $\begin{array}{c}\text { Observed } \\
\text { numbers }\end{array}$ & 5 & 13 & 20 & 13 & 51 \\
\hline \multirow{3}{*}{ (b) $\begin{array}{r}52, x y, t_{1} t_{2} \\
\times 53, x x, t_{2}\end{array}$} & & $51, t_{1} t_{2} t_{2}$ & $52, \mathrm{t}_{2} \mathrm{t}_{2}+52, \mathrm{t}_{1} \mathrm{t}_{2}$ & $53, t_{1}+53, t_{2}$ & 54 & \\
\hline & $\begin{array}{l}\text { Expected } \\
\text { numbers }\end{array}$ & $5 \cdot 25$ & $15 \cdot 75$ & $15 \cdot 75$ & $5 \cdot 25$ & 42 \\
\hline & $\begin{array}{c}\text { Observed } \\
\text { numbers }\end{array}$ & 10 & 12 & 16 & 4 & 42 \\
\hline \multirow{3}{*}{ (c) $\begin{aligned} & 52, x y, t_{1} t_{2} \\
& \times 53, x x, t_{3}\end{aligned}$} & & $51, t_{1}, t_{2} t_{3}$ & $\begin{array}{l}52, \mathrm{t}_{1} \mathrm{t}_{2}+52, \mathrm{t}_{1} \mathrm{t}_{3} \\
+52, \mathrm{t}_{2} \mathrm{t}_{3}\end{array}$ & $\begin{array}{l}53, t_{1}+53, t_{2} \\
+53, t_{3}\end{array}$ & 54 & \\
\hline & $\begin{array}{c}\text { Expected } \\
\text { numbers }\end{array}$ & $2 \cdot 875$ & $8 \cdot 625$ & $8 \cdot 625$ & 2.875 & 23 \\
\hline & $\begin{array}{c}\text { Observed } \\
\text { numbers }\end{array}$ & 2 & 5 & 12 & 4 & 23 \\
\hline Chromosome number & & 51 & 52 & 53 & 54 & \\
\hline \multirow[t]{2}{*}{ Total } & $\begin{array}{c}\text { Expected } \\
\text { number }\end{array}$ & $14 \cdot 5$ & $43 \cdot 5$ & $43 \cdot 5$ & $14 \cdot 5$ & 116 \\
\hline & $\begin{array}{c}\text { Observed } \\
\text { number }\end{array}$ & 17 & 30 & 48 & 21 & 116 \\
\hline
\end{tabular}

$\chi_{3}^{2}=$ not significant $(1: 3: 3: 1$ ratio $)$.

Table 6. Numbers of progeny with respective chromosome number, from previously unrecorded matings of translocation sheep

\begin{tabular}{|c|c|c|c|c|c|}
\hline \multirow{2}{*}{$\frac{\text { Matings }}{\text { (a) } 52, x y, t_{1} t_{1} \times 53, x x, t_{1}}$} & \multicolumn{4}{|c|}{$\begin{array}{c}\text { Numbers of progeny of each chromosome } \\
\text { type in parentheses }\end{array}$} & \multirow{2}{*}{$\frac{\text { Total }}{69}$} \\
\hline & & $52, \mathrm{t}_{1} \mathrm{t}_{1}(35)$ & $53, \mathrm{t}_{1}(34)$ & & \\
\hline (b) $52, x y, t_{2} t_{1} \times 52, x x, t_{1} t_{1}$ & & $52, \mathrm{t}_{1} \mathrm{t}_{1}(11)$ & & & 11 \\
\hline (c) $52, \mathrm{xy}, \mathrm{t}_{2} \times 52, \mathrm{xx}, \mathrm{t}_{2}$ & & $52, \mathrm{t}_{2} \mathrm{t}_{2}(4)$ & $53, \mathrm{t}_{2}(17)$ & $54(6)$ & 27 \\
\hline (d) $52, \mathrm{xy}, \mathrm{t}_{2} \mathrm{t}_{2} \times 52, \mathrm{xx}, \mathrm{t}_{2} \mathrm{t}_{2}^{\prime}$ & & $52, \mathrm{t}_{2} \mathrm{t}_{2}(5)$ & & & 5 \\
\hline (e) $52, \mathrm{xy}, \mathrm{t}_{2} \mathrm{t}_{2} \times 52, \mathrm{xx}, \mathrm{t}_{2}$ & & $52, \mathrm{t}_{2} \mathrm{t}_{2}(15)$ & $53, t_{2}(15)$ & & 30 \\
\hline (f) $52, \mathrm{xy}, \mathrm{t}_{2} \mathrm{t}_{2} \times 52, \mathrm{xx}, \mathrm{t}_{1} \mathrm{t}_{3}$ & & $52, t_{2} t_{x}(5)$ & & & 5 \\
\hline (g) $53, \mathrm{xy}, \mathrm{t}_{3} \times 54, \mathrm{xx}$ & & & $53, t_{3}(41)$ & $54(31)$ & 72 \\
\hline (h) $52, x y, t_{3} t_{3} \times 52, x x, t_{3} t_{3}$ & & $52, \mathrm{t}_{3} \mathrm{t}_{3}(7)$ & & & 7 \\
\hline (i) $52, x y, t_{3} t_{3} \times 52, x x, t_{1} t_{1}$ & & $52, \mathrm{t}_{1} \mathrm{t}_{3}(5)$ & & & 5 \\
\hline (j) $52, x y, t_{3} t_{3} \times 52, x x, t_{1} t_{2}$ & $51, \mathrm{t}_{1} \mathrm{t}_{2} \mathrm{t}_{3}(3)$ & $\begin{array}{l}52, \mathrm{t}_{1} \mathrm{t}_{3}(1) \\
52, \mathrm{t}_{2} \mathrm{t}_{\mathrm{x}}(1)\end{array}$ & & & 5 \\
\hline
\end{tabular}

Total 236 
Table 7. Reproductive details of mating translocation homozygous and double translocation heterozygous ewes to rams of different translocation karyotypes

\begin{tabular}{|c|c|c|c|c|c|c|}
\hline \multicolumn{3}{|c|}{ Mating category } & \multirow[b]{2}{*}{$\mathrm{E}_{\mathrm{M}}^{*}$} & \multirow[b]{2}{*}{$\mathrm{E}_{\mathrm{DM}}(\%)$} & \multirow[b]{2}{*}{$\mathrm{L}_{\mathrm{BM}}(\%)$} & \multirow[b]{2}{*}{$\mathrm{L}_{W M}(\%)$} \\
\hline & Ewes & Rams & & & & \\
\hline (a) & $52, \mathrm{xx}, \mathrm{t}_{1} \mathrm{t}_{1}$ & $\begin{array}{l}53, x y, t_{1}, 52, x y, t_{1} t_{1}, \\
52, x y, t_{3} t_{3}, 52, x y, t_{1} t_{2}, \\
52, x y, t_{1} t_{3}, 51, x y, t_{1} t_{2} t_{3}\end{array}$ & 52 & $5(9 \cdot 5)$ & $54(103 \cdot 8)$ & $46(88 \cdot 5)$ \\
\hline (b) & $52, \mathrm{xx}, \mathrm{t}_{2} \mathrm{t}_{2}$ & $\begin{array}{l}52, \mathrm{xy}, \mathrm{t}_{2} \mathrm{t}_{2}, 52, \mathrm{xy}, \mathrm{t}_{2} \mathrm{t}_{3}, \\
52, \mathrm{xy}, \mathrm{t}_{1} \mathrm{t}_{2} \mathrm{t}_{3}\end{array}$ & 9 & $0(0)$ & $10(111 \cdot 1)$ & $9(100 \cdot 0)$ \\
\hline (c) & $52, \mathrm{xx}, \mathrm{t}_{3} \mathrm{t}_{3}$ & $\begin{array}{l}53, x y, t_{1}, 52, x y, t_{1} t_{3}, \\
52, x y, t_{3} t_{3}, 51, x y, t_{1} t_{2} t_{3}\end{array}$ & 57 & $5(8 \cdot 8)$ & $69(121 \cdot 1)$ & $61(107 \cdot 0)$ \\
\hline (d) & $52, \mathrm{xx}, \mathrm{t}_{1} \mathrm{t}_{2}$ & $52, x y, t_{3} t_{3}, 51, x y, t_{1} t_{2} t_{3}$ & 46 & $5(10.9)$ & $48(104 \cdot 4)$ & $39(84 \cdot 8)$ \\
\hline (e) & $52, x x, t_{1} t_{3}$ & $52, x y, t_{2} t_{2}, 51, x y, t_{1} t_{2} t_{3}$ & 49 & $6(12 \cdot 2)$ & $55(112 \cdot 2)$ & $38(77 \cdot 8)$ \\
\hline (f) & $52, \mathrm{xx}, \mathrm{t}_{2} \mathrm{t}_{3}$ & $51, x y, t_{1} t_{2} t_{3}$ & 13 & $0(0)$ & $15(115 \cdot 4)$ & $14(107 \cdot 7)$ \\
\hline
\end{tabular}

$E_{M}=$ ewes mated; $E_{D M}=$ ewes dry to ewes mated, i.e. not producing a lamb; $L_{B M}=$ lambs born to ewes mated; $L_{\mathrm{wM}}=$ lambs weaned to ewes mated.

* Ewe lambs which did not mate were not included.

than at the start in 1969. Close inbreeding can particularly affect the performance of the ewe (Turner, 1969) and the survival of the progeny (Lax \& Brown, 1968). That this effect was becoming significant was suggested by the poor breeding performance of some individual ewes, which affected the result from the group. However, some ewes still continued to produce a high proportion of multiple births. For example one $52, x x, t_{1} t_{1}$ ewe mated over 5 years was dry in 2 years and produced single dead lambs in 3 years, while another $52, \mathrm{xx}, \mathrm{t}_{1} \mathrm{t}_{1}$ ewe was bred successfully as a ewe lamb and produced 9 live lambs in 5 matings. Similarly, one group of $852, x^{2}, t_{3} t_{3}$ ewes mated to a $51, \mathrm{xy}, \mathrm{t}_{1} \mathrm{t}_{2} \mathrm{t}_{3}$ ram in 1978 produced 14 lambs $\left(175 \% \mathrm{~L}_{\mathrm{wM}}\right)$.

Accepting the bias against the performance of the translocation sheep, the data from 118 homozygous ewes (Table 7), when combined, produced $98.3 \% \mathrm{~L}_{\mathrm{wM}}$ which was better than the New Zealand National Flock performance of $93.87 \% \mathrm{~L}_{\mathrm{wM}}$ for $1967-77$ and better than the performance of the commercial Romney flock husbanded under similar conditions at Massey

Table 8. Reproductive details of mating sheep heterozygous for multiple centric fusions and showing number of translocations of parents

\begin{tabular}{|c|c|c|c|c|c|c|c|}
\hline \multicolumn{3}{|c|}{ Mating category } & \multirow{2}{*}{$\begin{array}{c}\text { No. of } \\
\text { translocations } \\
\text { carried } \\
\text { heterozygously } \\
\text { by both } \\
\text { parents }\end{array}$} & \multirow[b]{2}{*}{$\mathrm{E}_{\mathrm{M}}$} & \multirow[b]{2}{*}{$\mathrm{E}_{\mathrm{DM}}(\%)$} & \multirow[b]{2}{*}{$\mathrm{L}_{\mathrm{BM}}(\%)$} & \multirow[b]{2}{*}{$\mathrm{L}_{\mathrm{wM}}(\%)$} \\
\hline & Rams & Ewes & & & & & \\
\hline (a) & $51, \mathrm{xy}, \mathrm{t}_{1} \mathrm{t}_{2} \mathrm{t}_{3}$ & $\begin{array}{l}54, \mathrm{xx}, 52, \mathrm{xx}, \mathrm{t}_{1} \mathrm{t}_{1}, \\
52, \mathrm{xx}, \mathrm{t}_{2} \mathrm{t}_{2}, 52, \mathrm{xx}, \mathrm{t}_{3} \mathrm{t}_{3}\end{array}$ & 3 & 54 & $1(1.85)$ & $62(114 \cdot 8)$ & $53(98 \cdot 1)$ \\
\hline (b) & $51, x y, t_{1} t_{2} t_{3}$ & $\begin{array}{l}53, \mathbf{x x}, \mathrm{t}_{1}, 53, \mathrm{xx}, \mathrm{t}_{2} \\
53, \mathrm{xx}, \mathrm{t}_{3}\end{array}$ & 4 & 126 & $13(10 \cdot 3)$ & $151(119 \cdot 8)$ & $128(101 \cdot 6)$ \\
\hline (c) & $51, x y, t_{1} t_{2} t_{3}$ & $\begin{array}{l}52, x x, t_{1} t_{2}, 52, x x, t_{2} t_{3}, \\
52, x x, t_{1} t_{3}\end{array}$ & 5 & 98 & $11(11 \cdot 2)$ & $104(106 \cdot 0)$ & $79(80 \cdot 6)$ \\
\hline (d) & $51, x y, t_{1} t_{2} t_{3}$ & $51, \mathbf{x x}, \mathrm{t}_{1} \mathrm{t}_{2} \mathrm{t}_{3}$ & 6 & 8 & $0(0)$ & $8(100 \cdot 0)$ & $8(100 \cdot 0)$ \\
\hline (e) & $52, x x, t_{1} t_{2}$ & $\begin{array}{l}53, \mathrm{xx}, \mathrm{t}_{1}, 53, \mathrm{xx}, \mathrm{t}_{2}, \\
53, \mathrm{xx}, \mathrm{t}_{3}\end{array}$ & 3 & 122 & $18(14 \cdot 8)$ & $140(114 \cdot 8)$ & $116(95 \cdot 1)$ \\
\hline
\end{tabular}


University, which was $89.01 \% \mathrm{~L}_{\mathbf{w M}}$. Similar comment could be made about the 108 double translocation heterozygous ewes which produced $84.25 \% \mathrm{~L}_{\mathrm{wM}}$. The latter figures reflected the high perinatal lamb loss of the 52,xx, $\mathrm{t}_{1} \mathrm{t}_{3}$ genotype (Table $10(\mathrm{e})$ ). The number of dry ewes from the combined data in Table 7 was $9.29 \%$ which is higher than the national average for Romney stud flocks (Quinlivan \& Martin, 1971).

Table 9. Reproductive details of mating translocation-carrying sheep with 49 and 50 chromosomes

\begin{tabular}{|c|c|c|c|c|c|c|}
\hline \multicolumn{2}{|c|}{ Mating category } & \multirow[b]{2}{*}{$\mathrm{E}_{\mathbf{M}}$} & \multirow[b]{2}{*}{$\mathrm{E}_{\mathrm{DM}}$} & \multirow[b]{2}{*}{$\mathrm{L}_{\text {BM }}(\%)$} & \multirow[b]{2}{*}{$\mathrm{L}_{\mathrm{wM}}(\%)$} & \multirow[b]{2}{*}{$\% \mathrm{C}_{\mathrm{R}}$} \\
\hline Rams & Ewes & & & & & \\
\hline (a) $50, x y, t_{1} t_{2} t_{3} t_{3}$ & $54, \mathrm{xx}$ & 20 & 1 & $24(120)$ & $24(120)$ & 90 \\
\hline (b) $49, x y, t_{1} t_{2} t_{2} t_{3} t_{3}$ & $\left.\begin{array}{l}51, \mathbf{x x}, \mathrm{t}_{1} \mathrm{t}_{2} \mathrm{t}_{3}(5) \\
51, \mathbf{x x}, \mathrm{t}_{1} \mathrm{t}_{2} \mathrm{t}_{\mathbf{x}}(2) \\
51, \mathbf{x x}, \mathrm{t}_{1} \mathrm{t}_{1} \mathrm{t}_{3}(1) \\
51, \mathbf{x x}, \mathrm{t}_{1} \mathrm{t}_{3} \mathrm{t}_{3}(1) \\
51, \mathbf{x x}, \mathrm{t}_{2} \mathrm{t}_{\mathbf{x}} \mathrm{t}_{\mathbf{x}}(3) \\
51, \mathbf{x x}, \mathrm{t}_{1} \mathrm{t}_{2} \mathbf{t}_{(4)} \\
51, \mathbf{x x}, \mathrm{t}_{2} \mathrm{t}_{3} \mathbf{t}_{\mathbf{x}}(3)\end{array}\right\}$ & 19 & 1 & $19(100)$ & $16(84 \cdot 2)$ & 89 \\
\hline (c) $49, x y, t_{1} t_{2} t_{2} t_{3} t_{3}$ & $\left.\begin{array}{r}50, x x, t_{1} t_{1} t_{2} t_{3}(1) \\
50, x x, t_{2} t_{2} t_{x} t_{x}(1) \\
50, x x, t_{1} t_{2} t_{2} t_{3}(2) \\
49, x x, t_{1} t_{1} t_{2} t_{3} t_{3}(1)\end{array}\right\}$ & 5 & 1 & $4(80)$ & $4(80)$ & 80 \\
\hline
\end{tabular}

$$
\% C_{R}=\% \text { of ewes conceiving to first service. }
$$

Table 10. Lamb losses analysed and compared between mating categories of Tables 7 and 8

Mating category

\begin{tabular}{|c|c|c|c|c|c|c|c|c|c|}
\hline & \multirow[b]{2}{*}{$\mathbf{E}_{\mathbf{M}}$} & \multirow[b]{2}{*}{$\mathbf{L}_{\mathbf{B}}$} & \multirow[b]{2}{*}{$\mathbf{L}_{B}-\mathbf{L}_{\mathbf{w}}$} & \multirow[b]{2}{*}{ Premature } & \multirow{2}{*}{$\begin{array}{l}\text { Perinatal } \\
\text { death }\end{array}$} & \multirow{2}{*}{$\begin{array}{l}\text { Perinatal } \\
\text { to weaning }\end{array}$} & \multirow[b]{2}{*}{ Comments } \\
\hline & Ewes & Rams & & & & & & & \\
\hline (a) & $52, \mathrm{xx}, \mathrm{t}_{1} \mathrm{t}_{1}$ & $\begin{array}{l}52, x y, t_{1}, 52, x y, t_{1} t_{1}, \\
52, x y, t_{3} t_{3}, 52, x y, t_{1} t_{2}, \\
52, x y, t_{1} t_{3}, 51, x y, t_{1} t_{2} t_{3}\end{array}$ & 52 & 54 & 8 & 0 & 8 & 0 & \\
\hline (b) & $52, \mathrm{xx}, \mathrm{t}_{2} \mathrm{t}_{2}$ & $\begin{array}{l}52, x y, t_{2} t_{2}, 52, x y, t_{2} t_{2}, \\
52, x y, t_{1} t_{2} t_{3}\end{array}$ & 9 & 10 & 1 & 0 & 1 & 0 & \\
\hline (c) & $52, \mathrm{xx}, \mathrm{t}_{3} \mathrm{t}_{3}$ & $\begin{array}{l}53, \mathrm{xy}, \mathrm{t}_{1}, 52, \mathrm{xy}, \mathrm{t}_{1} \mathrm{t}_{3}, \\
52, \mathrm{xy}, \mathrm{t}_{3}, \mathrm{t}_{3}, 51, \mathrm{xy}, \mathrm{t}_{1} \mathrm{t}_{2} \mathrm{t}_{3}\end{array}$ & 57 & 69 & 12 & 2 & 7 & 3 & $\begin{array}{l}\text { One ewe had no } \\
\text { milk in } 2 \text { lambings }\end{array}$ \\
\hline (d) & $52, x x, t_{1} t_{2}$ & $52, x y, t_{3} t_{3}, 51, x y, t_{1} t_{2} t_{3}$ & 46 & 48 & 9 & 1 & 7 & 1 & \\
\hline (e) & $52, x x, t_{1} t_{3}$ & $52, x y, t_{2} t_{2}, 51, x y, t_{1} t_{2} t_{3}$ & 49 & 55 & 17 & 0 & 16 & 1 & $\begin{array}{l}\text { One triplet born with } \\
\text { cleft palate }\end{array}$ \\
\hline (f) & $52, \mathrm{xx}, \mathrm{t}_{2} \mathrm{t}_{3}$ & $51, x y, t_{1} t_{2} t_{3}$ & 13 & 15 & 1 & 0 & 1 & 0 & \\
\hline (g) & $51, x y, t_{1} t_{2} t_{3}$ & $\begin{array}{l}54, \mathrm{xx}, 52, \mathrm{xx}, \mathrm{t}_{1} \mathrm{t}_{1}, \\
52, \mathrm{xx}, \mathrm{t}_{2} \mathrm{t}_{2}, 52, \mathrm{xx}, \mathrm{t}_{3} \mathrm{t}_{3}\end{array}$ & 54 & 62 & 9 & 0 & 9 & 0 & $\begin{array}{l}\text { One wry necked } \\
\text { lamb destroyed }\end{array}$ \\
\hline (h) & $51, x y, t_{1} t_{2} t_{3}$ & $\begin{array}{l}53, \mathrm{xx}, \mathrm{t}_{1}, 53, \mathrm{xx}, \mathrm{t}_{2} \\
53, \mathrm{xx}, \mathrm{t}_{3}\end{array}$ & 126 & 151 & 23 & 1 & 22 & 0 & $\begin{array}{l}\text { Included } 1 \text { set of trip- } \\
\text { lets all died at birth }\end{array}$ \\
\hline (i) & $51, x y, t_{1} t_{2} t_{3}$ & $\begin{array}{l}52, \mathrm{xx}, \mathrm{t}_{1} \mathrm{t}_{2}, 52, \mathrm{xx}, \mathrm{t}_{2} \mathrm{t}_{3}, \\
52, \mathrm{xx}, \mathrm{t}_{1} \mathrm{t}_{3}\end{array}$ & 98 & 104 & 25 & 1 & 21 & 3 & $\begin{array}{l}\text { One ewe with twins } \\
\text { died at birth }\end{array}$ \\
\hline (j) & $51, \mathrm{xy}, \mathrm{t}_{1} \mathrm{t}_{2} \mathrm{t}_{3}$ & $51, \mathrm{xx}, \mathrm{y}_{1} \mathrm{t}_{2} \mathrm{t}_{3}$ & 8 & 8 & 0 & 0 & 0 & 0 & \\
\hline \multirow[t]{2}{*}{ (k) } & $52, x y, t_{1} t_{2}$ & $\begin{array}{l}53, \mathrm{xx}, \mathrm{t}_{1}, 53, \mathrm{xx}, \mathrm{t}_{2}, \\
53, \mathrm{xx}, \mathrm{t}_{3}\end{array}$ & 122 & 140 & 24 & 2 & 22 & 0 & \\
\hline & Totals & & 634 & 712 & $129^{*}$ & 7 & 114 & 8 & \\
\hline
\end{tabular}

* Percentage of lambs dead $=18 \cdot 1$. 
Table 8 is of particular significance because it was possible that the ewes and rams of this group could have produced a high level of aneuploid gametes, which would have been reflected by a poor conception rate and an increased zygote loss as discussed previously. For this reason the number of translocations carried heterozygously among both parents are given and they indicate that, if the effects of non-disjunction were additive and aneuploid gametes were taking place in fertilization, a much poorer breeding performance should have occurred. However, in no instance was the conception rate lower than $78 \%$ (New Zealand national data $78 \cdot 8 \%$ for Romney stud flocks (Quinlivan \& Martin, 1971)), again suggesting that significant numbers of unbalanced zygotes were not being formed. Although the number of dry ewes was $10.29 \%$ for the whole group, in Groups 8(a) and 8(d) very low numbers of dry ewes were reported, which suggests that the higher than average dry ewe number was not associated with zygotic aneuploidy per se. The small number of animals in Group 8(d), in which ewes and rams were triple heterozygotes, should have shown a high level of embryonic loss reflected in poor conception rates if aneuploid gametes had been formed and participated in fertilization.

The data in Table 9 demonstrate the fertility of sheep with 49 and 50 chromosomes. The breeding data from all these animals derive from matings of 7-and 18-month-old ewes. Table 10 is a summary of the lamb losses of the mating groups described in Tables 7 and 8 and shows the relatively low 'genetic potential' of the animals used, due probably to the factors described previously. It also emphasizes that the main lamb loss was in the perinatal area, the majority of these losses being due to dystocia although the flock was very closely shepherded, as explained previously (Bruère, 1974a).

\section{Phenotypic anomalies}

In previous work with the tobacco mouse a decrease in testis size and spermatogenesis has been recorded (Cattanach \& Moseley, 1973; Cattanach, 1978). In the sheep odd anomalies such as hour-glass testes (Bruère \& Mills, 1971) and segmental aplasia (Bruère, Chapman \& Wyllie, 1972) and testicular hypoplasia in one ram (Long, 1978a; A. N. Bruère unpublished data) have been reported in translocation-carrying sheep. However, in the present study of 1995 progeny, less than $1 \%$ of obvious phenotypic abnormalities were observed. These included severely undershot jaw (1), wry neck (1), hour-glass testes (2), freemartin sheep (2), unilateral cryptorchids (6) and cleft palate (1). Several lambs were born showing signs of chorea which was diagnosed as Hairy Shaker disease which is caused by a transmissible agent.

\section{Discussion}

The extensive occurrence of centric fusions in domestic sheep has led, as for other species, to much speculation about their significance. The data presented here, when considered with previous reports (Bruère, 1974a, 1975; Bruère \& Chapman, 1974; Long, 1978b), give little or no reason to conclude that in sheep centric fusions are significantly deleterious in either the heterozygous or the homozygous condition. There is every reason to expect, from the wide variety of matings which took place, that there would have been some evidence of either a marked reduction in fertility or the development of some abnormal animals. In all classes of animals with translocations remarkably regular segregation products were recorded in their progeny. It would appear therefore that as in mice (White, Crandall, Raveche \& Tjio, 1978) the rapid combination of translocation does not significantly affect the random segregation process of the individual translocation heterozygotes.

As in previous reports of sheep carrying fewer translocation chromosomes, good conception rates and an average breeding performance were recorded. In no instance was there a serious fall in fertility as has been recorded in the $F_{1}$ of the tobacco mouse $\times$ domestic mouse (Gropp et al., 1970) and the Apennine mouse $\times$ laboratory mouse (Capanna et al., 1976). Pending the 
analysis of the triple heterozygotes $\left(\mathrm{t}_{1} \mathrm{t}_{2} \mathrm{t}_{3}\right)$ for levels of aneuploid secondary spermatocyte frequency, it would appear that although this may be shown to be higher than in single heterozygotes (Chapman \& Bruère, 1975) it does not lead to production of aneuploid zygotes. It should, however, be emphasized, that as in mice (White et al., 1978) the levels of aneuploidy in individual heterozygotes need not necessarily correlate with those of the combined heterozygotes, although in many examples an increase has been described (Gropp et al., 1970; Cattanach \& Moseley, 1973).

There is now little or no evidence to suggest that, in translocation-carrying sheep, aneuploid gametes are either formed or take place in fertilization and the previous conclusions (Bruère, 1974a, 1975; Bruère \& Chapman, 1974; Long, 1978a) of their elimination during spermatogenesis is even more strongly supported. Roosen-Runge (1973) suggested that cell loss in spermatogenesis is species specific and that in some species chromosomally abnormal cells are removed before maturation is complete. More recently Bunch \& Foote (1977) have reached a similar conclusion to explain the absence of aneuploid progeny from kara-tau (Argarli) Ovis ammon nigromontana $(2 \mathrm{n}=56) \times$ mouflon Ovis musimon $(2 \mathrm{n}=54)$ hybrids. In mice, for which there have been many reports of the formation of aneuploid zygotes, Baranov \& Dyban (1976) found one translocation-carrying strain with no evidence of aneuploid secondary spermatocytes which formed viable gametes.

The marked reduction in fertility reported in $F_{1}$ tobacco mouse $\times$ domestic mouse progeny are now concluded to be the result of the different genetic backgrounds of each species. In both cases the animals have been geographically isolated from each other and have developed some degree of reproductive isolation. In sheep, translocation carriers have not become geographically isolated and therefore no reproductive barriers could have developed. For reproductive barriers to evolve, a prolonged period of geographic isolation is essential (allopatric speciation) (Mayr, 1963). The number of reports from several countries of the unimpaired mating which takes place between different species of sheep with different chromosome numbers (Nadler, Lay \& Hassinger, 1971; Bunch, Foote \& Spillett, 1976; Y. Sharipov, personal communication) further supports this conclusion.

The absence of increased numbers of obvious phenotypic anomalies and the normal conception rates suggest that it is highly unlikely that embryos trisomic for any of the 3 translocations have either been formed or developed. This was confirmed in one study on $t_{1}$ blastocysts by Long (1977) which showed no evidence of trisomy in 102 13-18-day blastocysts and an equal segregation of $t_{1}$ and non-translocation homologues. In mice gross abnormalities attributable to trisomy resulting from the non-disjunction of translocation chromosomes have been reported frequently. White et al. (1974a, b) demonstrated a high incidence of early fetal death and cleft palate as a result of trisomy 19 in the laboratory mouse. Similarly, Gropp, Giers \& Kolbus (1974) produced mice trisomic for chromosome 4 with exencephaly and marked fetal hypoplasia.

The successful breeding of sheep with 50,49 and now 48 chromosomes confirms the remarkable stability of the sheep chromosomes. This is reflected in the cytological similarities of the chromosomes of bovids which in general have uniform $\mathrm{G}$ bands and $\mathrm{C}$ bands (Evans, Buckland \& Sumner, 1973; Buckland \& Evans, 1978a, b), and in four species, sheep (Ovis aries), cattle (Bos taurus), goat (Capra hircus) and aoudad (Ammotragus lervia), the nucleolar organizer regions (NORs) are homologous (Henderson \& Bruère, 1979). It now seems clear that the presence of at least 3 stable centric fusions (Robertsonian translocations) in domestic sheep, even in triply homozygous animals $(2 \mathrm{n}=48)$, is in no way detrimental to either the individual or the population. The mere persistence of such a polymorphic system both naturally for probably 100 years or more (Bruère et al., 1978) and experimentally gives weight to the argument that future investigation of such systems in domestic animals should be to determine their genetic meaning. In no way should they be eliminated arbitrarily from our animal populations on limited veterinary hypothesis. 
We gratefully acknowledge the assistance of $\mathrm{Mr} \mathrm{M}$. Ford, Mr M. Harris, Mr A. C. Bruère and Miss J. F. Bruère, with shepherding of the translocation flock; Mrs L. M. Henderson for identification of some of the sheep; Mrs V. Darroch for typing the manuscript; and the Wellcome Trust of Great Britain for financial support of this project.

\section{References}

Baranov, V.S. \& Dyban, A.P. (1976) Prezygotic selection of male gametes in laboratory mice. Tsitologiya 18, 556-559.

Bruère, A.N. (1974a) The segregation patterns and fertility of sheep heterozygous and homozygous for three different Robertsonian translocations. $J$. Reprod. Fert. 41, 453-465.

Bruère, A.N. (1974b) The discovery and biological consequences of some important chromosome anomalies in populations of domestic animals. In Proc. 1st Wld Congr. Genet. appl. Livestock Prod., Madrid, pp. 151-175. Graficas Orbe, S.L. Paddilla, 82 Madrid.

Bruère, A.N. (1975) Further evidence of normal fertility and the formation of balanced gametes in sheep with one or more different Robertsonian translocations. $J$. Reprod. Fert. 45, 323-331.

Bruère, A.N. \& Chapman, H.M. (1974) Double translocation heterozygosity and normal fertility in domestic sheep. Cytogenet. Cell Genet. 13, 342351.

Bruère, A.N. \& Mills, R.A. (1971) Observations on the incidence of Robertsonian translocations and associated testicular changes in a flock of New Zealand Romney sheep. Cytogenetics 10, 260-272.

Bruère, A.N., Chapman, H.M. \& Wyllie, D.R. (1972) Chromosome polymorphism and its possible implications in the select Drysdale breed of sheep. Cytogenetics 11, 233-246.

Bruère, A.N., Zartman, D.L. \& Chapman, H.M. (1974) The significance of the G-bands, and C-bands of three different Robertsonian translocations of domestic sheep (Ovis aries). Cytogenet. Cell Genet. 13, 479-488.

Bruère, A.N., Chapman, H.M., Jaine, P.M. \& Morris, R.M. (1976) Origin and significance of centric fusions in domestic sheep. J. Hered. 67, 149-154.

Bruère, A.N., Evans, E.P., Burtenshaw, M.O. \& Brown, B.B. (1978) Centric fusion polymorphism in Romney Marsh Sheep of England. J. Hered. 69, 8-10.

Buckland, R.A. \& Evans, H.J. (1978a) Cytogenetic aspects of phylogeny in the Bovidae. 1. G-banding. Cytogenet. Cell Genet. 21, 42-63.

Buckland, R.A. \& Evans, H.J. (1978b) Cytogenetic aspects of phylogeny in the Bovidae. II. C-banding Cytogenet. Cell Genet. 21, 64-71.

Bunch, T.D. \& Foote, W.C. (1977) Evolution of the $2 n=$ 54 Karyotype of domestic sheep (Ovis aries). Annls Génét. Sél. anim. 9, 509-515.

Bunch, T.D., Foote, W.C. \& Spillett, J.J. (1976) Translocations of acrocentric chromosomes and their implications in the evolution of sheep (Ovis). Cytogenet. Cell Genet. 17, 122-136.

Capanna, E., Gropp, A., Winking, H., Noack, G. \& Civitelli, M.V. (1976) Robertsonian metacentrics in the mouse. Chromosoma 58, 149-154.
Cattanach, B.M. (1978) Crossover suppression in mice heterozygous for tobacco mouse metacentrics. Cytogenet. Cell Genet. 20, 264-281.

Cattanach. B.M. \& Moseley, H. (1973) Nondisjunction and reduced fertility caused by the tobacco mouse metacentric chromosomes. Cytogenet. Cell Genet. 12, 264-287.

Chapman, H.M. \& Bruère, A.N. (1975) The frequency of aneuploidy in the secondary spermatocytes of normal and Robertsonian translocation-carrying rams. J. Reprod. Fert. 45, 333-342.

Evans, H.J., Buckland, R.A. \& Sumner, A.T. (1973) Chromosome homology and heterochromatin in goat, sheep and ox studied by banding techniques. Chromosoma 42, 383-402.

Gropp, A., Tettenborn, U. \& Von Lehmann, E. (1970) Chromosomenvariation von Robertson'schen Typus bei der Tabakmaus, M. Poschiavinus, und ihren Hybriden mit der Laboratoriumsmaus. Cytogenetics 9, 9-23.

Gropp, A., Giers, D. \& Kolbus, U. (1974) Trisomy in the fetal backcross progeny of male and female metacentric heterozygotes of the mouse. I. Cytogenet. Cell Genet. 13, 511-535.

Gustavsson, 1. (1969) Cytogenetics, distribution and phenotypic effects of a translocation in Swedish cattle. Hereditas 63, 68-169.

Henderson, L.M. \& Bruère, A.N. (1977) Association of nucieolus organizer chromosomes in domestic sheep (Ovis aries) shown by silver staining. Cytogenet. Cell Genet. 19, 326-334.

Henderson, L.M. \& Bruère, A.N. (1979) Conservation of nucleolus organizer regions during evolution in sheep, goat, cattle and aoudad. Can. J. Genet. Cytol. 21, $1-8$.

John, B. (1976) Chromosome change and evolutionary change. In Population Cytogenetics (Institute of Biology's Studies in Biology, No. 70.) Edward Arnold Ltd, London.

John, B. \& Freeman, M. (1975) Causes and consequences of Robertsonian exchange. Chromosoma 52, 123-136.

Kurnit, D.M., Brown, F.L. \& Maio, J.J. (1978) Mammalian repetitive DNA sequences in a stable Robertsonian system. Characterization, in situ hybridizations, and cross-species hybridizations of repetitive DNAs in calf, sheep and goat chromosomes. Cytogenet. Cell Genet. 21, 145-167.

Lax, J. \& Brown, G.H. (1968) The influence of maternal handicap, inbreeding, and ewe's bodyweight at 15-16 months of age on reproduction rate in Australian merinos. Aust. J. agric. Res. 19, 433-442.

Long, S.E. (1977) Cytogenetic examination of preimplantation blastocysts of ewes mated to rams heterozygous for the Massey I $\left(t_{1}\right)$ translocation. Cytogenet. Cell Genet. 18, 82-89. 
Long, S.E. (1978a) Chiasma counts and non-disjunction frequencies in a normal ram and in rams carrying the Massey I $\left(\mathrm{t}_{1}\right)$ Robertsonian translocation. J. Reprod. Fert. 53, 353-356.

Long, S.E. (1978b) Reproductive performance of ewes mated to rams heterozygous for the Massey $I\left(t_{1}\right)$ centric fusion (Robertsonian) translocation. Vet. Rec. 102, 399-401.

Mayr, E. (1963) Animal Species and Evolution. Harvard University Press, Cambridge, Massachusetts.

Nadler, C.F., Lay, D.M. \& Hassinger, J.D. (1971) Cytogenetic analysis of wild sheep populations in Northern Iran. Cytogenetics 10, 137-152.

Quinlivan, T.D. \& Martin, C.A. (1971) Survey observations on the reproductive performance of both Romney stud and commercial flocks throughout New Zealand. I. National Romney Stud performance. N.Z. J. agric. Sci. 14, 417-433.

Roosen-Runge, E.C. (1973) Germinal-cell loss in normal metazoan spermatogenesis. J. Reprod. Fert. 35, 339348.
Tettenborn, U. \& Gropp, A. (1970) Meiotic non-disjunction in mice and mouse hybrids. Cytogenetics 9, 272283.

Turner, H.N. (1969) Genetic improvement and reproduction rate in sheep. Anim. Breed. Abstr. 37, 545563.

White, B.J., Tjio, J.H., Van de Water, L.C. \& Crandall, C. (1974a) Trisomy 19 in the laboratory mouse. I. Frequency in different crosses at specific development stages and relationship of trisomy to cleft palate. Cytogenet. Cell Genet. 13, 217-231.

White, B.J., Tjio, J.H., Van de Water, L.C. \& Crandall, C. (1974b) Trisomy 19 in the laboratory mouse. II. Intra-uterine growth and histological studies of trisomics and their normal littermates. Cytogenet. Cell Genet. 13, 232-245.

White, B.J., Crandall, C., Raveche, E.S. \& Tjio, J.H. (1978) Laboratory mice carrying three pairs of Robertsonian translocations: establishment of a strain and analysis of meiotic segregation. Cytogenet. Cell Genet. 21, 113-138. 